\title{
Scalp Erosions that Do Not Heal: The Diagnostic Value of Trichoscopy
}

\author{
Matilde lorizzo $^{\text {a }}$ Werner Kempf ${ }^{\mathrm{b}}$ Lidia Rudnicka ${ }^{\mathrm{c}}$ \\ aPrivate Dermatology Practice, Bellinzona, Switzerland; ${ }^{b}$ Kempf und Pfaltz Histologische Diagnostik, \\ Zurich, Switzerland; ' Department of Dermatology, Medical University of Warsaw, Warsaw, Poland
}

\section{Question}

A 60-year-old man was referred for a 4 months history of a post-traumatic wound of the scalp that did not heal (Fig. 1). Physical examination revealed tender yellow hemorrhagic crusts on an erythematous and eroded base. There was a bigger involvement at the vertex and 2-small satellite erosions covered with crusts in both temporal areas. At dermoscopy, diffuse extravasation, dotted vessels, and the presence of anagen hairs covered by root sheaths were visible within the wound area (Fig. 2). The pull test was positive, especially in perilesional areas with mostly anagen hair roots. No symptoms were referred by the patient except a mild burning sensation when he tried to cover the scalp with a hat. A closer physical examination revealed small bleeding erosions also in the oral mucosa and in the nasal cavity epithelium.

At medical history, he referred a type 2 diabetes mellitus and a Helicobacter pylori-negative gastritis. No medication was taken by the patient except for oral antidiabetic drugs. Laboratory examinations (complete blood cells count, VES, PCR, liver/renal functions, thyroid function, immunodeficiency panel, syphilis panel, and

karger@karger.com

(c) 2021 S. Karger AG, Basel

www.karger.com/sad

Karger"

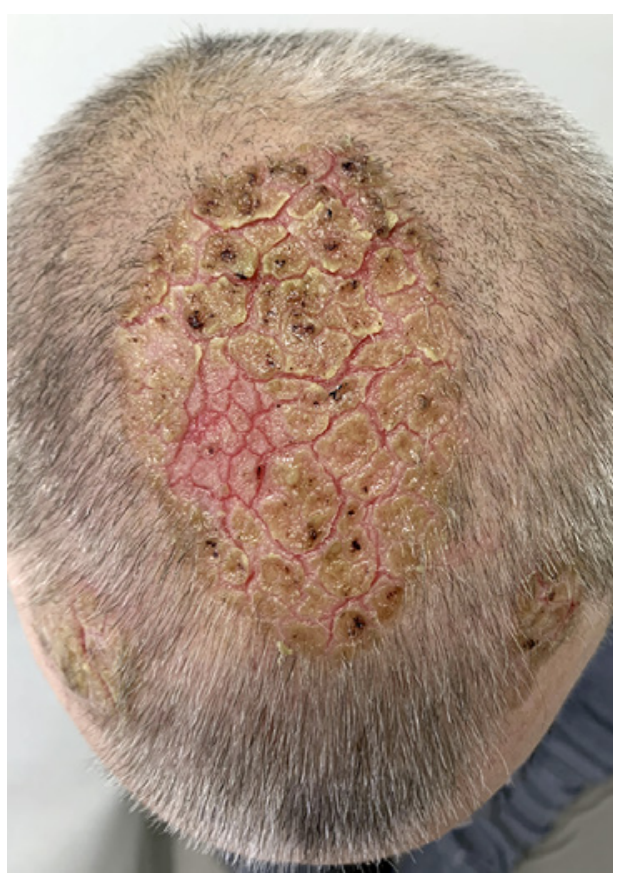

Fig. 1. Clinical presentation of the patient at diagnosis: tender yellow hemorrhagic crusts on an erythematous and eroded base were clearly visible. 


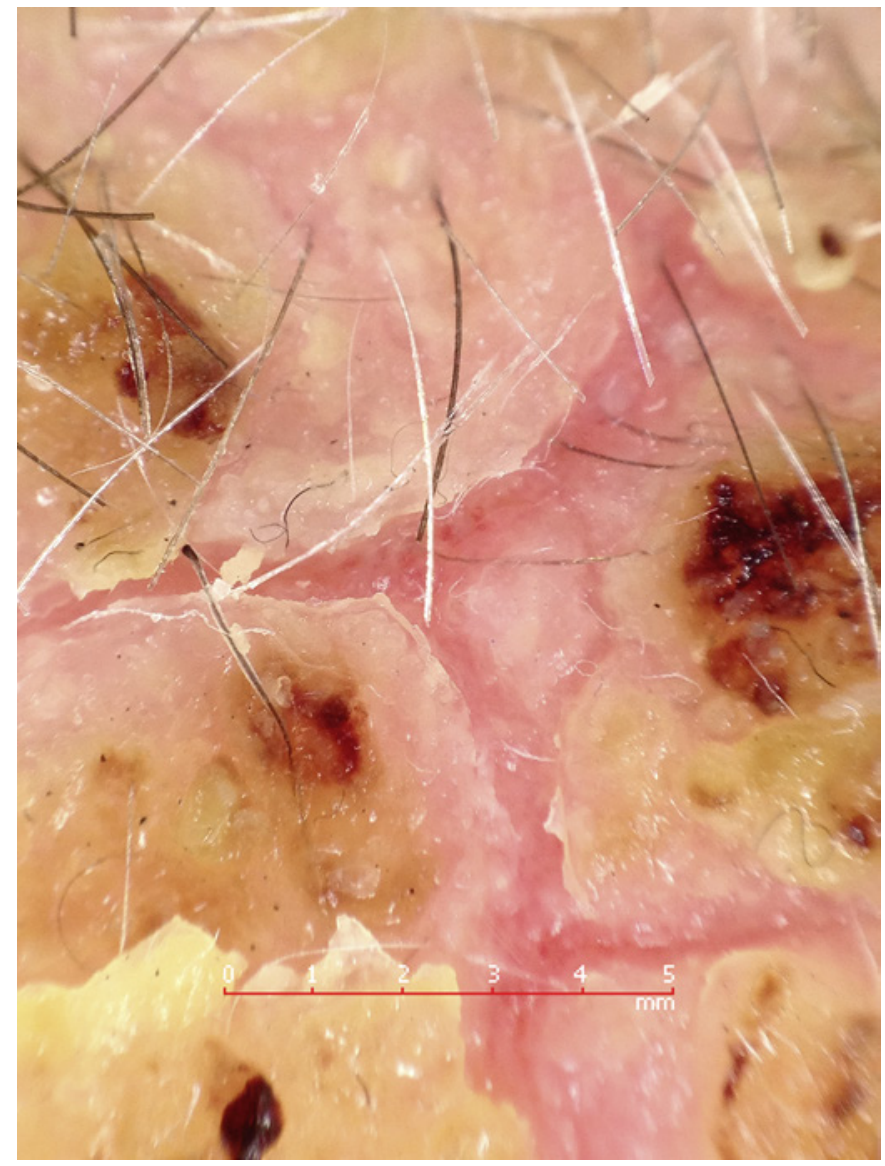

Fig. 2. At dermoscopy extravasation, dotted vessels and presence of anagen hairs covered by root sheaths were visible within the wound area.

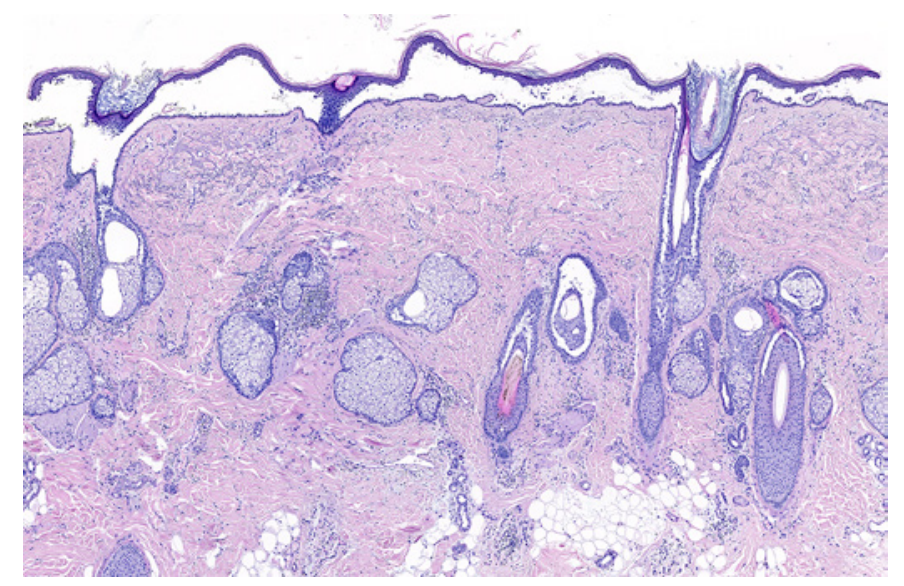

Fig. 3. Histopathological examination of scalp biopsy demonstrates intraepithelial blister with detachment of the upper parts of the epidermis.

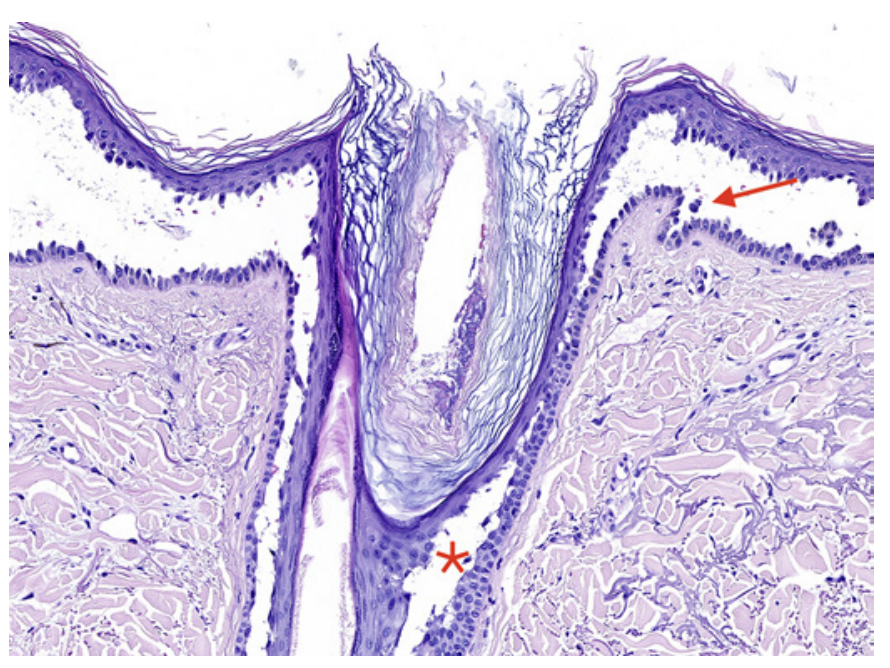

Fig. 4. Suprabasal acantholysis (arrow) was present in the interfollicular epidermis as well as in the hair follicle epithelia (asterisk).

epidermis as well as the hair follicle epithelia (Fig. 4 asterisk). The basal layer with palisaded keratinocytes (tombstone appearance) formed the bottom of the blister. A perivascular perifollicular lymphohistiocytic infiltrate with admixture of plasma cells was present in the upper dermis.

What is your diagnosis? 


\section{Answer}

Based on these findings a diagnosis of pemphigus vulgaris was made. A confirmation biopsy was also taken from the oral mucosa. Anti-desmoglein 1 (Dsg1) and anti-desmoglein 3 (Dsg3) IgG antibodies were both elevated but not the anti-bullous pemphigoid (BP180 and 230) and collagen VII ones. The patient was also checked for a paraneoplastic syndrome (serological markers, gastroscopy, pancolonoscopy, and abdomen CT scan), but no abnormality was found. Due to a difficultly to manage diabetes, the patient started rituximab $1 \mathrm{~g} / 100$ $\mathrm{mL}$ and kept steroids only as a local treatment $(0.05 \%$ clobetasol propionate cream overnight once every 2 days and perilesional injections of $10 \mathrm{mg} / \mathrm{mL}$ triamcinolone acetonide once a month).

Scalp erosions have been observed in patients with pemphigus vulgaris, and the involvement of the oral mucosa is a well-known feature of the disease. According to the literature, scalp involvement is the first location in around $10 \%$ of cases [1]. Besides erosions, patchy alopecia and anagen shedding are also a possible presentation of the disease in the scalp area $[2,3]$.

Intraepidermal blisters, and subsequent erosions, due to loss of intercellular cohesion are due to IgG antibodies that bind primarily Dsg3, and less often Dsg1, expressed on keratinocytes. Dsg1 and 3 are expressed also in anagen hair follicles, and the anagen shedding is likely due to acantholysis between outer root sheath keratinocytes $[4,5]$.

As suggested by other authors [6] we believe that, in our patient, trauma was the responsible for the development of pemphigus. It has been suggested, in fact, that injuries that disturb the dermoepidermal junction might express or enhance epidermal pemphigus antigens and lead to epitope spreading in genetically predisposed patients. In the absence of a trauma, these patients are unable to develop the disease. Moreover, the healing process itself and a possible inflammation due to a bacterial infection may constitute an obstacle to the normal differentiation of keratinocytes, making cells more vulnerable even to low IgG $[7,8]$.

Pemphigus vulgaris should always be considered in the differential diagnosis of poorly healing wounds. A presumptive diagnosis can be made through dermoscopy or hair samples prior to incisional biopsy, but histopathology confirmation is always mandatory. Scalp dermoscopy and trichoscopy are a valid aid to identify the right place to biopsy: typical features are extravasation and vascular abnormalities like dotted vessels with whit- ish halo, circular vessels, and polymorphic vessels [9, 10]. A direct immunofluorescence test can also be made on hair samples as a complementary exam: in presence of pemphigus vulgaris, IgG deposition in intercellular spaces of outer root sheaths keratinocytes can, in fact, be easily detectable.

\section{Statement of Ethics}

The patient's consent was obtained for the pictures and eventual publication.

\section{Conflict of Interest Statement}

The authors have no conflicts of interest to declare.

\section{Funding Sources}

The authors did not receive any funding.

\section{Author Contributions}

Every author listed meets the qualifications for authorship and has had the opportunity to read and comment upon the submitted manuscript.

\section{Keywords}

Scalp disorders · Pemphigus vulgaris · Erosive pustular dermatosis of the scalp - Dermoscopy - Trichoscopy 


\section{References}

1 Sar-Pomian M, Konop M, Gala K, Rudnicka L, Olszewska M. Scalp involvement in pemphigus: a prognostic marker. Postepy Dermatol Alergol. 2018;35(3):293-8.

2 Delmonte S, Semino MT, Parodi A, Rebora A. Normal anagen effluvium: a sign of pemphigus vulgaris. Br J Dermatol. 2000;142(6): 1244-5.

3 Xie D, Bilgic-Temel A, Abu Alrub N, Murrell DF. Alopecia in autoimmune blistering diseases: a systematic review of pathogenesis and clinical features of disease. Skin Appendage Disord. 2019;5(5):263-75.
$4 \mathrm{Wu} \mathrm{H}$, Stanley JR, Cotsarelis G. Desmoglein isotype expression in the hair follicle and its cysts correlates with type of keratinization and degree of differentiation. J Invest Dermatol. 2003;120(6):1052-7.

5 Veraitch O, Ohyama M, Yamagami J, Amagai M. Alopecia as a rare but distinct manifestation of pemphigus vulgaris. J Eur Acad Dermatol Venereol. 2013;27(1):86-91.

6 Daneshpazhooh M, Fatehnejad M, Rahbar Z, Balighi K, Ghandi N, Ghiasi M, et al. Traumainduced pemphigus: a case series of 36 patients. J Dtsch Dermatol Ges. 2016;14(2):166-71.
7 Ruocco V, Pisani M. Induced pemphigus. Arch Dermatol Res. 1982;274(1-2):123-40.

8 Hameed A, Khan AA. Koebner phenomenon in pemphigus vulgaris. Br J Dermatol. 1996; 135(1):152-3.

9 Sar-Pomian M, Kurzeja M, Rudnicka L, Olszewska $M$. The value of trichoscopy in the differential diagnosis of scalp lesions in pemphigus vulgaris and pemphigus foliaceus. An Bras Dermatol. 2014;89(6):1007-12.

10 Sar-Pomian M, Rudnicka L, Olszewska M. Trichoscopy: a useful tool in the preliminary differential diagnosis of autoimmune bullous diseases. Int J Dermatol. 2017;56(10):9961002. 